\title{
Domestic swine in a visceral leishmaniasis endemic area produce antibodies against multiple Leishmania infantum antigens but apparently resist to L. infantum infection
}

\author{
Evandro Moraes-Silva a,b, Fabiana Rodrigues Antunes a , Marcio Silva Rodrigues a , \\ Fred da Silva Julião ${ }^{b}$, Artur Gomes Dias-Lima ${ }^{a}$, Valderez Lemos-de-Sousa ${ }^{a}$, \\ Adriano Costa de Alcantara ${ }^{\mathrm{b}}$, Eliana Almeida Gomes Reis ${ }^{\mathrm{a}}$, \\ Maria Nakatani ${ }^{\mathrm{c}}$, Roberto Badaró ${ }^{\mathrm{c}}$, Mitermayer Galvão Reis ${ }^{\mathrm{a}}$, \\ Lain Pontes-de-Carvalho ${ }^{a}$, Carlos Roberto Franke ${ }^{\text {b,* }}$ \\ ${ }^{a}$ Centro de Pesquisas Gonçalo Moniz (CPqGM), Fundação Oswaldo Cruz, Rua Waldemar Falcão 121, Brotas, \\ CEP: 40295-001, Salvador, Bahia, Brazil \\ ${ }^{\mathrm{b}}$ Laboratório de Infectologia Veterinária, Escola de Medicina Veterinária, Universidade Federal da Bahia, \\ Av. Adhemar de Barros 500, Ondina, CEP: 40.170-110, Salvador, Bahia, Brazil \\ ${ }^{c}$ Laboratório de Doenças Tropicais, Hospital Universitário Professor Edgard Santos, Universidade Federal da Bahia, \\ Rua João das Botas s/n, Canela, CEP: 40110-160, Salvador, Bahia, Brazil
}

Received 11 May 2005; received in revised form 20 March 2006; accepted 12 April 2006

\begin{abstract}
In order to investigate whether pigs can be infected by Leishmania infantum, a serological and parasitological study was carried out on swine in the Jequié municipality, Northeast of Brazil. Anti-Leishmania infantum antibodies were detected in 37 out of 92 swine (40.2\%), by two different assays: an anti-L. infantum lysate and an anti-K39 recombinant protein ELISA. An experimental study was also carried out to verify the susceptibility of domestic pigs to $L$. infantum infection. Three sows inoculated with $10^{8}$ stationary-phase infective $L$. infantum promastigotes ( $26 \%$ metacyclic promastigotes) per kilogram of body weight produced antiLeishmania antibodies until the end of the experiment, 11 months later. No parasites, however, could be visualized through optical microscopy of spleen, liver and bone marrow or by in vitro culture of these organs. Homogenates of these organs were also inoculated in hamsters, without producing infection. No Leishmania DNA was detected by polymerase chain reaction (PCR) in sand flies fed on these animals. The results indicate that domestic pigs bitten by $L$. infantum-infected vectors in the endemic area do not display a full infection pattern, and the positive association in endemic areas between the presence of swine and infection in canines may not be ascribable to the former acting as a parasite reservoir.
\end{abstract}

(C) 2006 Published by Elsevier B.V.

Keywords: Visceral leishmaniasis; Pig; Immune response; Experimental infection

\footnotetext{
* Corresponding author. Tel.: +557133315042 ; fax: +557133315042 .

E-mail address: franke@ufba.br (C.R. Franke).
}

\section{Introduction}

American visceral leishmaniasis (AVL) is defined as a zoonotic disease caused by Leishmania infantum, although it was considered to be caused by the Leish- 
mania chagasi in the New World. This knowledge has been taxonomically challenged over the last years by the absence of genetic differences between $L$. chagasi and $L$. infantum using the available molecular tools to compare both species (Mauricio et al., 1999). Thus, in this paper, $L$. infantum is going to be used throughout the text instead of $L$. chagasi. From a public health perspective, AVL is of significant concern (Desjeux, 2004). In Brazil, the disease occurs in dry, sparsely forested areas, mainly in the Northeast, where a high percentage of the population lives in poverty (WHO, 1992; Franke et al., 2002a). This already undesirable situation, however, is gradually worsening: AVL is spreading to other areas, including large cities and their peripheries (Jerônimo et al., 1994; Cunha et al., 1995; Mathis and Deplazes, 1995; Franke et al., 2002b). Although there are several indirect lines of evidence incriminating dogs as the main reservoir of L. infantum (Nicolle, 1908), this is still a matter of controversy (Dietze et al., 1997; Paranhos-Silva et al., 1998; Ashford et al., 1998). In fact, information on the extent to which other domestic animals may participate in transmitting $L$. infantum is scarce, and in particular, there is practically no data on the role of swine in this process.

Pigs are often present in the vicinity of houses in endemic areas with high prevalence of AVL (Sherlock, 1996; Moreira et al., 2003). In such areas, these animals are often raised under conditions that are far from optimal: in back yards, or even being permitted to wander freely through the streets. They seem to attract more Phlebotominae sand flies, the L. infantum insect vectors, than canines and humans (Morrison et al., 1993). Moreover, they are easily bitten by these insects (Ferro et al., 1997), which puts them at risk for being infected by Leishmania sp. and, eventually, serving as parasite reservoirs. This is suggested by the observation that the presence of swine in a given area increased the risk of canine infection by $L$. infantum by a 4.1 -fold factor (IC 95\% 1.2-13.8; $p=0.02$ ) (Moreira et al., 2003). The reason for this, however, is not clear. In addition to the possibility that pigs may constitute a $L$. infantum reservoir, pigsties may create multiplying environments for the AVL sand fly vector, since swine may both readily feed the female sand flies and release organic compounds into the soil that could serve as food for sand fly larvae (Moreira et al., 2003). Indeed, in Colombia, 11 Lutzomyia longipalpis sand fly larvae were found near pigsties in an AVL endemic focus (Ferro et al., 1997).

The hypothesis that pigs are susceptible to the disease received a boost after a Leishmania-infected pig was found in an area of Northeastern Brazil where cutaneous Leishmaniasis is endemic. Moreover, the high numbers of amastigotes found in the infected pig's cutaneous lesion indicated that it could act as a source of infection for sand flies (Brazil et al., 1987).

The study described here examined the prevalence of anti-Leishmania antibodies in the swine population of a Brazilian town where AVL is endemic. The possibility of pigs being infected by L. infantum was also investigated.

\section{Materials and methods}

\subsection{Study area}

The study was conducted in an area of high AVL incidence, the Jequié municipality. An endemic community in the Northeast of Brazil, Jequié has about 200,000 inhabitants. The municipality is located between a forest zone and scrub savanna, in the geographic coordinates of $13^{\circ} 52^{\prime} \mathrm{S}$ and $40^{\circ} 4^{\prime} \mathrm{W}$.

\subsection{Sample collection}

Blood samples were collected from 92 freely bred swine of different ages, during two separate trips to the endemic area. In 36 of these animals, an ear skin fragment was removed and in 28, a hepatic aspirate was collected concomitantly with the blood collection. The aspirates were done with the introduction of a $40-\mathrm{mm} \times 12$ $\mathrm{mm}$ needle, connected to a $20-\mathrm{ml}$ syringe, in the penultimate or in the last but two left intercostals spaces. Part of the aspirate was used to make glass slide smears and the remaining was immediately placed onto an ice box for transportation, before being cultivated at $24{ }^{\circ} \mathrm{C}$ in NNN medium (Lainson and Shaw, 1979), about $4 \mathrm{~h}$ later.

Bone marrow aspirations and removal of spleen fragments were performed during necropsy, in only two seropositive animals.

Sera from 28 swine, raised under the same conditions as those from the high-prevalent endemic area, were obtained in the region of Santo Amaro, Bahia, where human AVL cases are very sporadic (Franke et al., 2002b). These sera were used as an internal negative control, based on the fact that this region has similar environmental and economical characteristics when compared to the Jequié municipality but few or no cases of leishmaniasis. Thirty sera obtained from pigs raised in a confined environment in Santa Catarina State, a Leishmaniasis-free area, were used as negative controls.

\subsection{ELISA, sodium dodecil sulphate polyacrylamide gel electrophoresis (SDS-PAGE) and Western blotting}

ELISA was performed to detect antibodies against $L$. infantum, using as antigen a $L$. infantum promastigote 
lysate (anti-L. infantum ELISA) (Reed et al., 1990) or the K39 recombinant protein (anti-rK39 ELISA) (Burns et al., 1993).

The sera were diluted at 1:100. In both assays, a 1:10,000-diluted rabbit anti-pig IgG antibodyperoxidase conjugate was used (Sigma Chemical Company, St. Louis, USA).

The cut-off value was the mean optical density obtained with the serum samples of the 19 swine from the non-endemic area plus three standard deviations of the mean. The cut-off was 0.476 for the anti-L. infantum ELISA, and 0.595 for the anti-rK39 ELISA. In order to estimate the anti-Leishmania antibody seroprevalence, the confidence interval was determined, considering, for mathematical effects, an infinite population of swine, and the following equation was used:

$p \pm v \sqrt{\frac{p(1-p)}{n}}$,

where, $p$, proportion of positive animals; $v$, mathematical constant $=2.58 ; n$, size of sample tested $=92$.

The two-way ANOVA test was used for statistical analysis of the ELISA results.

Western blotting was done as described elsewhere (Qu et al., 1994) using an L. infantum lysate as antigen, 1:100-diluted pig sera, 1:5000-diluted anti-pig IgG-peroxidase conjugate and diaminobenzidine as the chromogen.

\subsection{Search for parasites}

Skin biopsy imprints and liver, spleen and bone marrow aspirate smears were fixed in glass slides.

Skin, liver and bone marrow samples were aseptically sowed in a two-phase culture medium (NNN medium plus liver-triptose infusion) (Lainson and Shaw, 1979), cultured at $24^{\circ} \mathrm{C}$ and examined weekly, by optical microscopy, for 5 weeks.

\subsection{Experimental inoculation of $L$. infantum, xenodiagnosis and assay for Leishmania DNA}

Three weaning sows, weighing about $10 \mathrm{~kg}$, bred in confinement and fed with a balanced diet, negative for antibodies against $L$. infantum in the assays described above, with fecal examinations negative for the presence of gastro-intestinal parasites, were intravenously inoculated with $10^{8}$ stationary-phase MHOM/BR2000/MERIVALDO L. infantum promastigotes (Paranhos-Silva et al., 2003) per kilogram of body weight which contained $2.6 \times 10^{8}$ Leishmania metacyclic promastigotes (Soares et al., 1993; Paranhos-Silva et al., 2003). Two hamsters were inoculated with this same batch of Leishmania, in order to serve as a control for parasite infectivity. The sows were monitored daily for fever and clinical abnormalities during the first 2 months after inoculation. After that, the animals were examined monthly up to 1 year after inoculation, when they underwent necropsy. Sera and skin samples were collected for 10 months and subjected to the assays for antibodies or to the parasite search described above. Xenodiagnosis with sand flies was carried out monthly after inoculation. Thirty L. longipalpis females, bred in our research facilities, were allowed to feed on glabrous areas of the sedated animals (the ear and the internal part of the thigh), during $30 \mathrm{~min}$. The presence of Leishmania in the sand flies was investigated by optical examination of their digestive tract contents, 4 days after feeding.

In addition, homogenates from liver, spleen and bone marrow tissues, collected for 10 months beginning 2 months after the inoculation, were direct examined for the presence of parasite in Giemsa-stained microscope slide smears and also inoculated in hamsters and sowed in culture medium, in an attempt to isolate Leishmania from these animals.

In order to bring about polymerase chain reactions (PCR), DNA was extracted from bone-marrow aspirates using the $G F X^{\mathrm{TM}}$ Genomic Blood DNA Purification Kit (Amersham Pharmacia Biotech Inc., Piscataway, USA) and the following synthetic oligonucleotides were used to amplify the conserved region of the kDNA minicircles: primer A: $5^{\prime}-(\mathrm{G} / \mathrm{C})(\mathrm{G} / \mathrm{C})(\mathrm{C} / \mathrm{G}) \mathrm{CC}(\mathrm{A} / \mathrm{C}) \mathrm{CTAT}$ (A/T) TTA CAC CAA CCCC; and primer B: 5'-CCG CCC CTA TTT TAC ACC AAC CCC following an adapted protocol from Schubach et al. (1998). This PCR has been shown to be able to detect the presence of DNA from one parasite (data not shown).

\section{Results}

Thirty-eight of the 92 (41.3\%) animals from the highprevalent AVL endemic area had anti- $L$. infantum antibodies, as detected by the anti-L. infantum ELISA, and 47 of the $92(51.1 \%)$ had antibodies detectable in the anti-rK39 ELISA (Fig. 1) and this difference was statistically significant (two-way ANOVA test, $p<0.05$ ). Thirty-seven sera $(40.2 \%)$ were positive in both assays, and $48(52.1 \%)$ were positive in at least one of the assays. The prevalence of animals with anti-Leishmania antibodies in the swine population of the Jequié municipality varied between 27.02 and $53.38 \%$ over the period of our sample, considering as positive animals with antibodies detectable in both assays, or between 38.69 and $65.51 \%$, considering as positive those with antibodies detectable in at least one assay. 


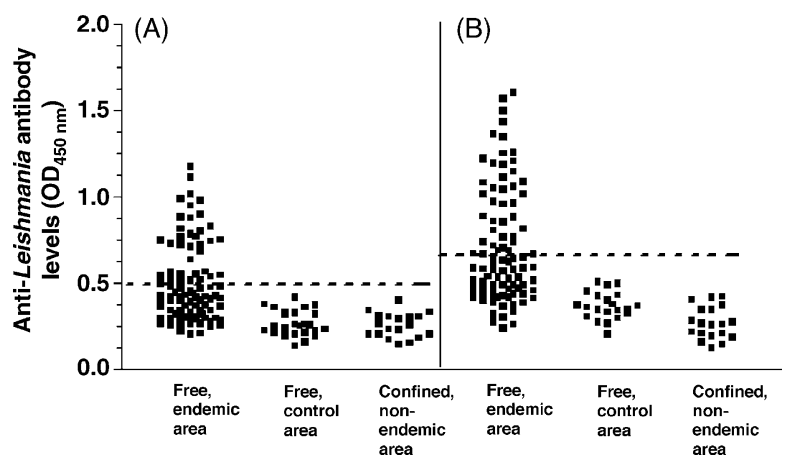

Fig. 1. Anti-L. infantum antibodies in pigs from an American visceral leishmaniasis endemic area. Antibodies were detected in ELISAs using as antigen a $L$. infantum promastigote lysate (A) or the K39 recombinant antigen (B). Animals were raised free in a high-prevalence AVL endemic area (free, endemic area) or in a socio-economically and geographically similar area (free, control area), and confined in a non-endemic area (confined, non-endemic area). Each symbol corresponds to the result obtained from an individual animal. The dotted lines indicate the values of the assay cut-offs.
The sera from 20 swine, with anti-Leishmania antibodies detectable by ELISA, recognized 3-14 antigens of apparent molecular weights varying from 20 to $205 \mathrm{kDa}$ (Fig. 2(a and b)). Five sera from swine originated from a control non-endemic area, did not recognize any band, and five sera from swine of the high-prevalent endemic area, negative in the ELISA, recognized zero to three bands (Fig. 2(a and c)).

Amastigote forms of Leishmania were not found in the microscopic analysis of the 198 samples (derived from 72 skin Giemsa-stained imprints, 56 liver Giemsastained smears, 25 bone-marrow stained smears), obtained from a total of 36 animals. No promastigote growth was observed in cultures of material removed from these animals.

The animals seemed healthy during the experiment, showing just a slight increase in body temperature in the first 3 months after the L. infantum inoculation. No amastigote forms were found in the microscopic analysis
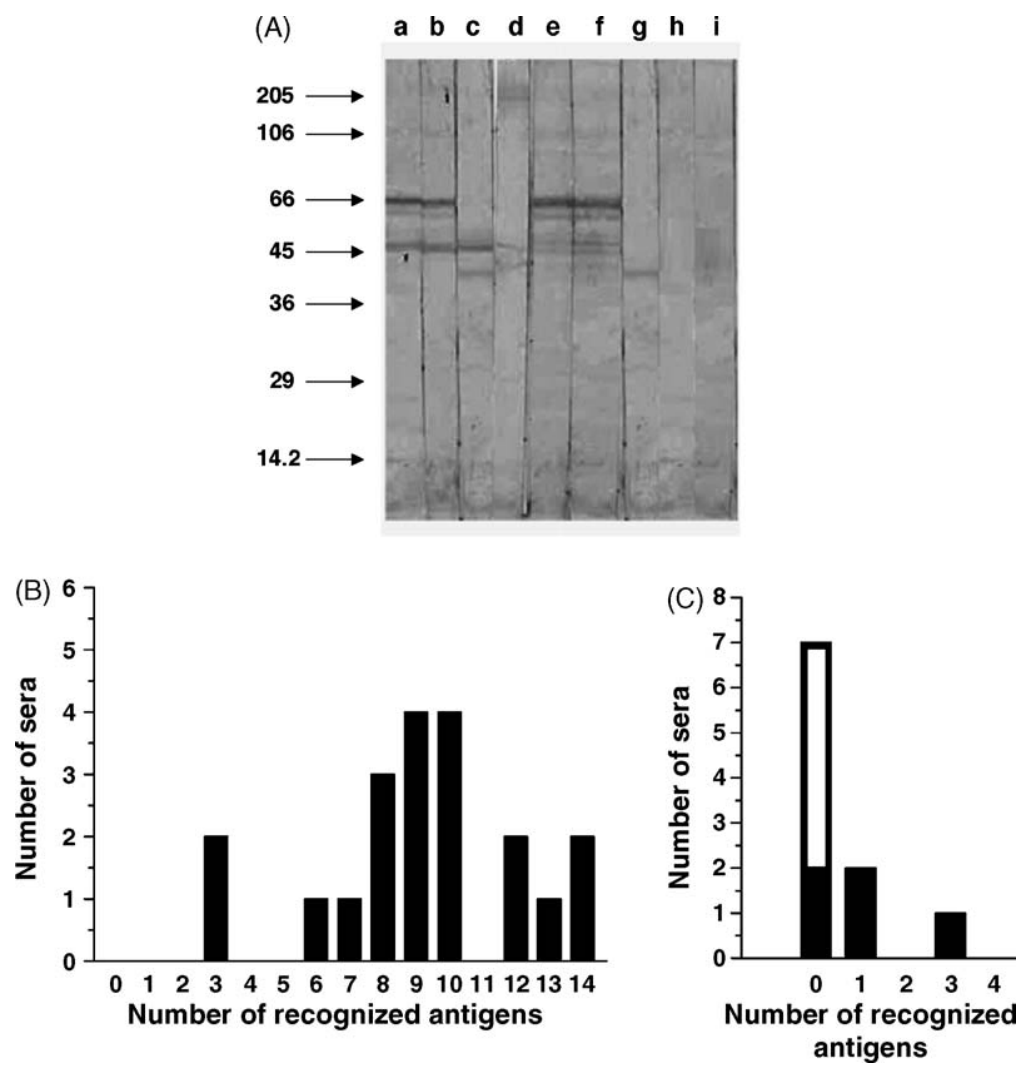

Fig. 2. Recognition of different $L$. infantum antigens by swine sera, from an endemic area for human and canine visceral leishmaniasis, in a Western blot of promastigote lysate. In (A) columns from (a-f) correspond to Western blot nitrocellulose strips incubated with swine sera with anti-L. infantum antibodies detectable by ELISA, and columns from ( $\mathrm{g}-\mathrm{i}$ ) correspond to strips incubated with sera from swines without detectable anti- $L$. infantum antibodies. The positions of the molecular weight markers are indicated by arrows. In (B) each column represents the number of sera recognizing, in the Western blot, the number of antigens indicated in the abscissa. Sera were from 20 swines with anti-Leishmania antibodies detectable by ELISA. In (C), the same is shown for 10 control sera, negative for anti-Leishmania antibodies in the ELISA (five of swines from the endemic area and five of swines from a non-endemic area). The sera from the non-endemic area are represented by the open area in the column. 

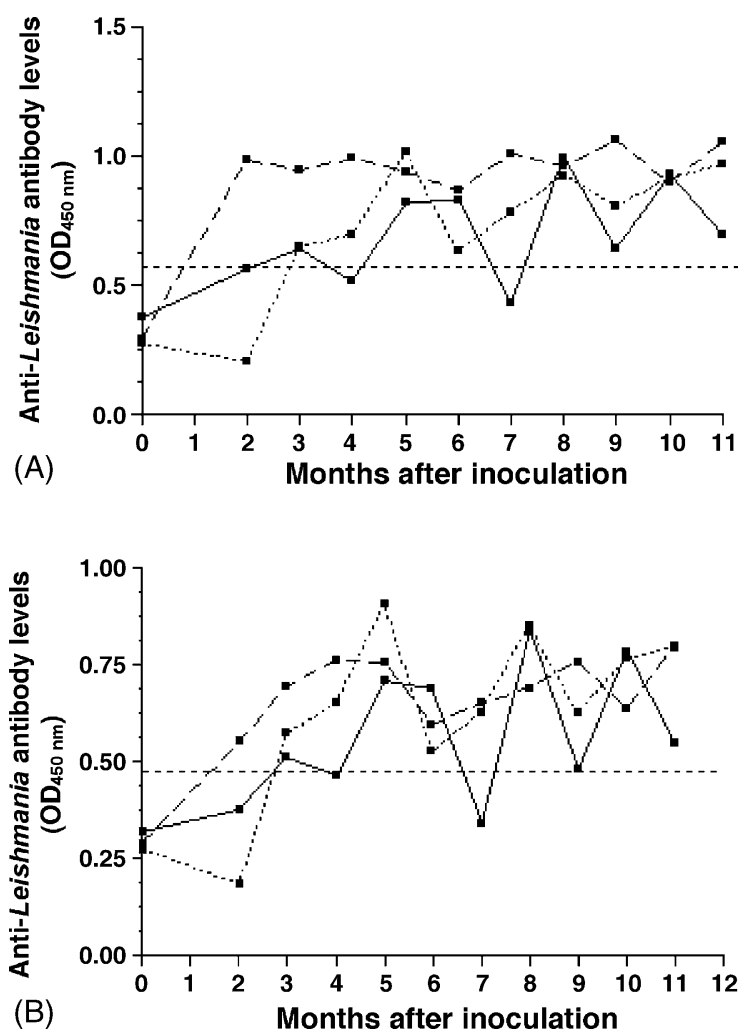

Fig. 3. Anti-Leishmania humoral immune responses in swines inoculated with $L$. infantum. Antibodies were detected in ELISAs using as antigen a $L$. infantum promastigote lysate (A) or the K39 recombinant antigen (B). Each curve represents the results obtained from an individual animal. The dotted line indicates de cut-off values.

of stained smears of liver, spleen and bone marrow. No promastigote forms were found in cultures of material from these organs. Hamsters inoculated with these materials, culled 6 months later, were negative for Leishmania. No flagellates were observed by optical microscopy in the digestive tract of sand flies that were allowed to feed on swine. No Leishmania DNA was identified in bone-marrow material by a PCR reaction that readily detected the positive control sample (not shown). At the third month after inoculation, there was a significant increase in anti-Leishmania antibody levels, as detected by ELISA, in the sera of the two animals. These levels were maintained, with some variation in two of the three animals, during the period of the experiment (Fig. 3).

\section{Discussion}

The present report is the first comprehensive investigation of the possible involvement of pigs in the epidemiology of zoonotic visceral leishmaniasis.
The high frequency of anti-Leishmania antibodies in the pigs of an endemic area, described here, suggests a previous contact by these animals with the parasite. Another possibility is that their antibodies could have been elicited by another infectious agent bearing crossreactive antigens. In a similar study, high levels of antiLeishmania donovani antibodies were found in domestic animals (oxen, donkeys and goats), also without detection of parasites (Mukhtar et al., 2000).

The utilization of the K39 recombinant antigen in the present study makes it less probable that the detected anti- $L$. infantum antibodies could be due to a crossreactive immune response. Anti-rK39 antibodies are practically undetectable in sera from human patients with tuberculosis, toxoplasmosis and malaria, in which false positive reactions occur in immunoassays using crude Leishmania antigens (Burns et al., 1993; Badaró et al., 1996). Regarding microorganisms most phylogenetically related to Leishmania, the rK39 antigen is also very specific as can be noted when antibodies from Trypanossoma cruzi-infected patients, or from cutaneous leishmaniasis patients, do not recognize it (Burns et al., 1993; Qu et al., 1994; Singh et al., 1995).

However, the possibility of a cross-reaction between the rK39 antigen and other pathogens that infect swine in the endemic area cannot be excluded. The absence of anti-Leishmania lysate and anti-rK39 antibodies in pigs from an area where AVL has been only sporadically reported and the animals are maintained in apparently the same conditions of those in the high-prevalent area, argues against this possibility. In addition, since several polypeptides with different molecular weights are recognized by antibodies from the animals (18 out of 20 tested animals recognized at least six different peptides) it is highly improbable that these antibodies could be the result of a cross-reaction between Leishmania and a phylogenetically related microorganism. The antigens recognized in our study are similar, in terms of molecular weights, to those found through reactivity with human (Pontes-de-Carvalho et al., unpublished data) and canine (Qu et al., 1994) sera.

The data obtained from experimental infection show that the Sus scrofa domesticus respond to the inoculation of Leishmania by producing circulating antibodies. The presence of antibodies until the end of the experiment, 11 months after inoculation, suggests that a subclinical infection is maintained, intense enough to keep the immune system stimulated, but remaining below the detection threshold of the parasitological methods used in the present study. Similar results have been reported in cats (Kirkpatrick et al., 1984). 
Despite the high frequency of anti-Leishmania antibodies and the diversity of the antibodies produced (shown by Western blot), verified in the swine samples from the high-prevalent endemic area studied here, further investigation of the origin of these antibodies is still necessary to determine whether they result from a previous contact with Leishmania or from a cross-reactive immune response. The findings that: (1) pigs respond to the inoculation of live Leishmania producing antibodies, as reported herein; (2) pigs are bitten by Phlebotaminae sand flies in an endemic area (Ferro et al., 1997; Moreira et al., 2003); and (3) Phlebotaminae sand flies in an endemic area transmit Leishmania to mammals (Deane and Deane, 1962; Lainson and Shaw, 1979; Sherlock, 1996), taken together, suggest that the first hypothesis is correct. In any case, notwithstanding the result of future investigations, swine do not seem to be an important reservoir for $L$. infantum, at least for the Jequié municipality, since, in the study described in the present report, it was not possible to detect the parasite by any of the techniques used in the examined animals. The absence of amastigote forms in the skin of the swine particularly suggests that such animals may not be a significant source of infection by L. infantum for the insect vector, although other researchers found numerous Leishmania amastigotes in one pig (Brazil et al., 1987), but without being able to further characterize the species. Dr. Reginaldo Peçanha Brazil told us that his team was not able to further characterize the Leishmania sp. from the pig, but they considered the morphological aspects similar to the Leishmania braziliensis and, some time after that, one child at the same house developed a limb skin ulcer from which they could isolate and further characterize by isoenzyme analysis as L. braziliensis (unpublished data, personal communication). Our findings are reinforced by the results obtained from the experimental inoculation of L. infantum in three sows, in which no parasites could be isolated. The results presented here provide the first evidence of the relationship of pigs in the epidemiology of urban/peri-urban AVL. Based on our data, swine do not seem to act as a reservoir of $L$. infantum but, at least in the Jequié municipality, they probably play an important role on the attraction and/or maintenance of the insect vector in the peridomicile area.

\section{Acknowledgements}

We would like to thank the Fundação Oswaldo Cruz-FIOCRUZ and the Conselho Nacional de Desenvolvimento Científico e Tecnológico-CNPq (CNPq $521130 / 98 \mathrm{NV}$ ) for their support of this study.

\section{References}

Ashford, A.D., David, J.R., Freire, M., David, R., Sherlock, I., Eulálio, M.C., Sampaio, D.P., Badaró, R., 1998. Studies on control of visceral leishmaniasis: impact of dog control on canine and human visceral leishmaniasis in Jacobina, Bahia, Brazil. Am. J. Trop. Med. Hyg. 59, 53-57.

Badaró, R., Benson, D., Eulálio, M.C., Freire, M., Cunha, S., Netto, E.M., Pedral-Sampaio, D., Madureira, C., Burns, J.M., Houghton, R.L., David, J.R., Reed, S.G., 1996. rK39: a cloned antigen of Leishmania infantum that predicts active visceral leishmaniasis. J. Infect. Dis. 173, 758-761.

Brazil, R.P., Nascimento, M.D.S.B., Macau, R.P., 1987. Infecção natural de um porco (Sus scrofa) por Leishmania em foco recente de leishmaniose tegumentar na ilha de São Luís. Maranhão. Mem. Inst. Oswaldo Cruz 82, 145

Burns Jr., J.M., Shreffler, W.G., Bensor, D.R., Ghalib, H.W., Badaró, R., Reed, S.G., 1993. Molecular characterization of a kinesinrelated antigen of Leishmania infantum that detects specific antibody in African and America visceral leishmaniasis. Proc. Natl. Acad. Sci. 90, 775-791.

Cunha, S., Freire, M., Eulalio, C., Cristovão, J., Netto, E., Johnson Junior, W.D., Reed, S.G., Badaró, R., 1995. Visceral leishmaniasis in a new ecological niche near a major metropolitan area of Brazil. Trans. R. Soc. Trop. Med. Hyg. 89, 155-158.

Deane, L.M., Deane, M.P., 1962. Visceral leishmaniasis in Brazil: geographical distribuition and transmission. Rev. Inst. Med. Trop. São Paulo 4, 198-212.

Desjeux, P., 2004. Leishmaniasis: current situation and new perspectives. Comp. Immunol. Microbiol. Infect. Dis. 27 (5), 305-318.

Dietze, R., Barros, G.B., Teixeira, L., Harris, J., Michelson, K., Falqueto, A., Corey, R., 1997. Effect of eliminating seropositive canines on the transmission of visceral leishmaniasis in Brazil. Clin. Infect. Dis. 25, 1240-1242.

Ferro, C., Prado, R., Torres, M., Morrison, A.C., 1997. Larval microhabitats of Lutzomyia longipalpis (Díptera: Psychodidae) in an endemic focus of visceral leishmaniasis in Colombia. J. Med. Entomol. 34, 719-728.

Franke, C.R., Ziller, M., Staubach, C., Latif, M., 2002a. Impact of the El Nino/Southern Oscillation on visceral leishmaniasis, Brazil. Emerg. Infect. Dis. 8 (9), 914-917.

Franke, C.R., Staubach, C., Ziller, M., Schlüter, H., 2002b. Trends in temporal and espatial distribuition of visceral and cutaneous leishmaniasis in state of Bahia, Brazil, from 1985 to 1999. Trans. R. Soc. Trop. Med. Hyg. 96, 236-241.

Jerônimo, S.M.B., Oliveira, R.M., Mackay, S., Costa, K.G., Sweet, J., Nascimento, E.T., Luz, K.G., Fernandes, M.S., Jerningan, J., Pearson, R.D., 1994. An urban outbreak of visceral leishmaniasis in Natal, Brazil. Trans. R. Soc. Trop. Med. Hyg. 88, 386-388.

Kirkpatrick, E.C., Farrell, J.P., Goldschmid, M.H., 1984. Leishmania infantum and L. Donovani: experimental infections in domestic cats. Exp. Parasitol. 58, 125-131.

Lainson, R., Shaw, J.J., 1979. The role of animals in the epidemiology of south America leishmaniasis. In: Lumsden, W.H.R., Evans, D.A. (Eds.), Biology of the Kinetopllastida, vol. 2. Academic Press, London, pp. 1-116.

Mathis, A., Deplazes, P., 1995. PCR and in vitro cultivation for detection of Leishmania spp. in diagnostic samples for humans and dogs. J. Clin. Microbiol. 33 (5), 1145-1149.

Mauricio, I.L., Howard, M.K., Stothard, J.R., Miles, M.A., 1999. Genomic diversity in the Leishmania donovani complex. Parasitology 119 (Part 3), 237-246. 
Moreira Jr, E.D., Souza, V.M.M., Sreenivasan, M., Lopes, N.L., Silva, R.B.B., Carvalho, L.P., 2003. Peridomestic risk factors for canine leishmaniasis in urban dwelling: new findings from a prospective study in Brazil. Am. J. Trop. Med. Hyg. 63, 393-397.

Morrison, A.C., Ferro, C., Tesh, R.B., 1993. Host preferences of the sand fly Lutzomyia longipalpis at an endemic focus of American visceral leishmaniasis in Colombia. Am. J. Trop. Med. Hyg. 49 (1), 68-75

Mukhtar, M.M., Sharief, A.H., El Saffi, S.H., Harith, A.E., Higazzi, T.B., Adam, A.M., Abdalla, H.S., 2000. Detection of antibodies to Leishmania donovani in a kala-azar endemic region in eastern Sudan: a preliminary report. Trans. R. Soc. Trop. Med. Hyg. 94, 33-36.

Nicolle, C., 1908. Origine canine du kala-azar infantile. Bull. Soc. Pathol. Exot. 1, 299-301.

Paranhos-Silva, M., Nascimento, E.G., Melro, M.C.B.F., Oliveira, G.G.S., Santos, W.L.C., Pontes-De-Carvalho, L.C., Oriveira-DosSantos, A.J., 1998. Cohort study on canine emigration and Leishmania infection in an endemic area for visceral leishmaniasis. Implications for the disease control. Acta Trop. 69, 75-83.

Paranhos-Silva, M., Oliveira, G.G., Reis, E.A., de Menezes, R.M., Fernandes, O., Sherlock, I., Gomes, R.B., Pontes-de-Carvalho, L.C., dos-Santos, W.L., 2003. A follow-up of Beagle dogs intradermally infected with Leishmania chagasi in the presence or absence of sand fly saliva. Vet. Parasitol. 114 (2), 97-111.
Qu, J.Q., Zhong, L., Masoom-Yasinzai, M., Abdur-Rab, M., Asku, H.S.K., Reed, S.G., Chang, K.P., 1994. Serodiagnosis of Asian leishmaniasis with a recombinant antigen from the repetitive domain of a Leishmania Kinesin. Trans. R. Soc. Trop. Med. Hyg. $88,543-545$.

Reed, S.G., Shreffler, V.G., Burms Jr., J.M., Scott, J.M., Orge, Mda.G., Ghalib, H.W., Siddig, M., Badaro, R., 1990. An improved serodiagnostic procedure for visceral leishmaniasis. Am. J. Trop. Med. Hyg. 43, 632-691.

Schubach, A., Haddad, F., Oliveira-Neto, M.P., Degrave, W., Pirmez, C., Grimaldi Jr., G., Fernandes, O., 1998. Detection of Leishmania DNA by polimerase chain reaction in scars of treated human patients. J. Infec. Dis. 178, 911-914.

Sherlock, I.A., 1996. Ecological interactions of visceral leishmaniasis in the State of Bahia, Brasil. Mem. Inst. Oswaldo Cruz 91, 671683.

Singh, S., Gilman-Sachs, A., Chang, K.P., Reed, S.G., 1995. Diagnostic and prognostic value of K39 recombinant antigen India Leishmaniasis. J. Parasitol. 81, 1000-1003.

Soares, N.M., Carvalho, E.M., Pinho, R.T., Pontes de Carvalho, L.C., 1993. Induction of complement-sensitivity in Leishmania amazonensis metacyclic promastigotes by protease treatment but not by specific antibodies. Parasitol. Res. 79, 340-342.

WHO, 1992. Information on the epidemiology and control of the Leishmaniasis by country or territory. pp. 47 . 